Revue de droit comparé du travail et de la sécurité sociale

2| 2018

Harcèlement moral au travail en jurisprudence

comparée

\title{
« Et soudain, ce silence entre nous ». Histoires de dialogues interrompus entre les Cours nationales et supranationales
}

Silvia Borelli et Marco Rocca

\section{OpenEdition}

Journals

Édition électronique

URL : https://journals.openedition.org/rdctss/1955

DOI : $10.4000 /$ rdctss. 1955

ISSN : 2262-9815

Éditeur

Centre de droit comparé du travail et de la sécurité sociale

Édition imprimée

Date de publication : 1 juin 2018

Pagination : $96-109$

ISSN : 2117-4350

Référence électronique

Silvia Borelli et Marco Rocca, « «Et soudain, ce silence entre nous». Histoires de dialogues interrompus entre les Cours nationales et supranationales », Revue de droit comparé du travail et de la sécurité sociale [En ligne], 2 | 2018, mis en ligne le 01 novembre 2021, consulté le 13 novembre 2021. URL : http://journals.openedition.org/rdctss/1955; DOI : https://doi.org/10.4000/rdctss. 1955

\section{(c) $(1)$}

Revue de droit comparé du travail et de la sécurité sociale est mise à disposition selon les termes de la Licence Creative Commons Attribution - Pas d'Utilisation Commerciale - Pas de Modification 4.0 International. 


\title{
SILVIA BORELLI
}

Professeur en droit du travail à l'Université de Ferrare

\section{MARCO ROCCA}

Chercheur post-doctoral à l'Université Hasselt, Chargé de cours à l'Université de Liège et Maître de conférences invité à l'Université Libre de Bruxelles - Solvay Business School

\section{« ET SOUDAIN, CE SILENCE ENTRE NOUS »}

\section{HISTOIRES DE DIALOGUES INTERROMPUS}

\author{
ENTRE LES COURS NATIONALES ET SUPRANATIONALES
}

\section{RÉSUMÉ}

La protection internationale des droits sociaux a fait l'objet d'un regain d'intérêt au cours des années de crise financière. Cela est dû à de multiples facteurs, notamment la réticence de la Cour européenne de justice à contrôler les restrictions aux droits sociaux fondamentaux découlant des mesures d'austérité. Cependant, l'impact réel et le suivi des décisions rendues par les organes de suivi au niveau international restent largement inexplorés. L'article se concentre sur deux exemples spécifiques, tirés des expériences italienne et belge, pour évaluer dans quelle mesure la protection internationale des droits sociaux a un impact au niveau national. Dans le contexte italien, l'affaire FIAT montre que l'intervention du Comité de I'OIT sur la liberté syndicale n'a apporté aucune amélioration effective des droits syndicaux, en raison de problèmes de procédure et de la valeur incertaine de la convention de I'OIT dans l'ordre juridique national. Du côté belge, les auteurs examinent l'application faite par les juges nationaux d'une décision du Comité européen des droits sociaux, critiquant l'habitude de concéder des injonctions provisoires interdisant les activités de piquetage à l'occasion d'une grève. En l'absence d'une intervention de la Cour suprême, les auteurs soutiennent que l'impact de la décision du CEDS reste confiné au rôle de loi non contraignante. Les auteurs concluent que la protection à plusieurs niveaux des droits fondamentaux doit être considérée comme un mécanisme puissant et pourtant fragile, où tous les rouages doivent tourner dans la bonne direction pour que l'ensemble puisse fonctionner : lorsque l'un de ces rouages s'arrête, l'ensemble du mécanisme s'arrête brusquement.

MotS-CLÉs : Droits fondamentaux, protection à plusieurs niveaux, droit international du travail.

\section{ABSTRACT}

The international protection of social rights has been the subject of a renewed interest during the years of the financial crisis. This is due to multiple factors, notably, the reticence of the European Court of Justice to scrutinise the restrictions to fundamental social rights brought about by austerity measures. However, the actual impact and follow up of decisions delivered by monitoring bodies at international level remains largely unexplored. The essay focuses on two specific examples, drawn from the Italian and Belgian experiences, to assess to what extent the international protection of social rights has an impact at national level. In the Italian context, the FIAT case shows that the intervention of the ILO Committee on freedom of association has not brought any effective improvement of trade union rights, due to procedural problems and the uncertain value of ILO convention in the national legal order. On the Belgian side, the authors consider the application made by national judges of a decision of the European Committee of Social Rights criticising the habit of conceding interim injunctions forbidding picketing activities in the occasion of a strike. Lacking an intervention of the Supreme Court, the authors argue that the impact of the decision by the ECSR remains confined to the role of soft law. The authors conclude that the " multilevel » protection of fundamental right should be considered as a powerful and yet fragile mechanism, where all the cogwheels have to turn in the right direction for it to function: when one of these cogwheels stops, the entire mechanism grinds to a halt.

KEYWORDS: Fundamental Rights; Multilevel Protection; International Labour Law. 
a protection multi-niveaux des droits sociaux a fait un retour en force dans les débats juridiques, tout au long des années de crise ${ }^{1}$. Parallèlement, le sens même du concept de multi-niveaux s'est amplifié. Auparavant, si l'attention portait essentiellement sur le recours au juge communautaire, la réticence (pour recourir à un euphémisme) de ce dernier à sanctionner les restrictions apportées aux droits fondamentaux résultant de la gestion de la crise par les institutions européennes l'a souvent rendu superflu.

Les acteurs juridiques et sociaux se sont ainsi retrouvés privés des protections proposées par le droit communautaire et ont dû, en conséquence, élargir leurs horizons respectifs pour chercher un point d'appui permettant de préserver les droits sociaux des attaques du rouleau compresseur de l'austérité. Ceci a conduit à raviver de nouveau l'attention sur le rôle des organes de contrôle des conventions de l'Organisation internationale du travail (OIT), de la Charte sociale européenne (CSE) et de la Convention européenne des droits de l'Homme (CEDH).

Le faible impact des décisions des organes supranationaux que l'on constate après avoir analysé la dite " jurisprudence de la crise » nous a convaincus de l'importance du fait de ne pas se limiter à l'analyse des décisions supranationales en tant que telles, même si ces dernières sont favorables et rassurantes mais, au contraire, d'en étudier la réémergence au niveau national et d'en vérifier l'impact réel sur la jurisprudence nationale.

Examiner le suivi des décisions des Cours supranationales signifie non seulement surveiller l'impact d'une décision particulière sur un cas concret mais, plus généralement, contrôler également l'impact d'une décision de la Cour supranationale sur le système juridique national. Etant donné que les Cours supranationales interprètent et appliquent des sources supranationales, il s'agit, en dernier lieu, de vérifier l'effet de ces sources sur le droit interne ${ }^{2}$. Sont-elles connues et utilisées par les Cours nationales ? À quelles fins ? Pour justifier ad adjuvandum une décision de la Cour nationale ? Pour interpréter le droit interne conformément à la source supranationale? Pour ne pas appliquer une disposition de droit interne qui ne serait pas conforme à la source supranationale ? Pour régler un cas qui n'est pas régi par le droit interne? En tant que critère d'appréciation dans le contrôle de la légitimité constitutionnelle des lois nationales?

1 A. Supiot, «Qui garde les gardiens ? La guerre du dernier mot en droit social européen », Semaine sociale Lamy, 2016, supplément $n^{\circ} 1746$, p. 1 ; F. Valdés Dal-Ré, «El constitucionalismo laboral europeo y la protección multinivel de los derechos laborales fundamentales: luces y sombras », 2016,

http://comunicacion.uva.es/export/sites/comunicacion/ documentos/Leccion-Fernando-Valdes.pdf

C. Kilpatrick, B. De Witte, «A comparative framing of fundamental rights challenges to social crisis measures in the Eurozone », European Journal of Social Law, 2014, p. 1.

2 Voir pour la législation de I'OIT X. Beaudonnet (dir.) Droit international du travail et droit interne. Manuel de formation pour les juges, juristes et professeurs de droit, Turin, Centre international de formation de l'OIT, 2009, p. 17 et s. ; pour la CSE, C. Benelhocine C., La Charte sociale européenne, Strasbourg, Éditions du Conseil de l'Europe, 2011, p. 94 et s. 
L'examen du suivi des décisions des Cours supranationales apparaît encore plus important à l'heure où ces Cours débattent entre elles. Comme cela a été expressément affirmé par le Comité européen des droits sociaux (C.E.D.S ci-après-décision portant sur la réclamation collective $n^{\circ} 85 / 2012, \S 74$ ), les principes et les normes constituant le système juridique de l'Union ne coïncident pas nécessairement avec le système de valeurs, de principes et de droits garantis par la Charte sociale européenne (CSE ci-après) et par les conventions de I'OIT.

La tension entre les Cours supranationales se répercute sur les juges nationaux qui, lorsqu'ils doivent trancher un cas concret, sont forcés de résoudre les contradictions existant entre les sources supranationales. La solution retenue face à ces contradictions diffère d'un État à l'autre : celle-ci dépend en effet de l'effet juridique reconnu à chacune des sources supranationales dans l'ordre interne et des règles de droit interne régissant les contradictions entre les normes. Donc, les Cours nationales sont tenues de trouver, dans leur propre système de référence, une solution aux conflits qui surviennent dans les États simultanément membres de plusieurs organisations internationales ${ }^{3}$.

Toutes les questions précitées mériteraient un examen bien plus approfondi que celui que l'on peut effectuer ici. Nous nous bornerons donc à présenter deux affaires explicitant quelques-uns des points faibles du dialogue multi-niveaux entre les Cours. Ces affaires concerneront deux pays très différents : d'une part, l'Italie, à savoir un État où une réflexion sérieuse sur l'effet juridique des conventions de I'OIT et de la CSE fait défaut - tant et si bien que ces sources sont quasiment ignorées par les tribunaux nationaux ou, pire encore, citées de manière erronée ou de façon à en limiter l'effet juridique ; d'autre part, la Belgique, pays où la CSE est connue des Cours mais où il n'existe aucun organe garantissant une interprétation et une application sans ambiguïté de cette dernière.

\section{I - LE CAS FIAT : LE CONTEXTE}

Les évènements syndicaux qui, depuis 2010, ont affecté le plus grand constructeur automobile italien ont été largement étudiés par la doctrine italienne ${ }^{4}$. En bref, Fiat a mis en œuvre une stratégie directe visant à expulser de ses usines la FIOM (Fédération des employés et ouvriers de la métallurgie de la Confédération général italienne du travail, CGIL), c'est-à-dire le syndicat qui compte (et qui comptait) le plus grand nombre d'adhérents au sein de l'entreprise. À cette fin, Fiat a mis fin aux accords syndicaux en vigueur et en juin 2011, elle a fait savoir qu'elle quittait Federmeccanica (Fédération des entreprises de la métallurgie) à compter du $1^{\text {er }}$ janvier 2012. Dès lors, l'unique convention collective en vigueur au sein des usines du groupe Fiat était la convention de groupe, signée par plusieurs syndicats mais pas par la FIOM. Étant donné que jusqu'à l'arrêt n 231/2013 de la Cour constitutionnelle ${ }^{5}$, l'article 19 du Statut des travailleurs permettait uniquement aux syndicats signataires de la convention collective applicable dans l'entreprise d'avoir

3 C. Panzera, "Diritti ineffettivi? Gli strumenti di tutela della carta sociale europea », Rivista AIC $n^{\circ} 1 / 2017$, p. 18.

4 Pour une reconstitution détaillée de l'affaire, voir A. Lassandari, « L'azione giudiziale come forma di autotutela collettiva », Lavoro e Diritto, 2014, p. 309 et F. Focareta, «II modello FCA-CNH, le parole e le cose », DLRI, 2017, p. 365.

5 Dans l'arrêt $n^{\circ} 231 / 2013$, la Cour constitutionnelle italienne a déclaré que les syndicats qui ont participé à la négociation collective peuvent créer leur RSE, alors même qu'ils n'ont pas signé la convention collective. 
des représentants syndicaux d'entreprise (RSE ci-après), la FIOM - le syndicat qui avait (et a encore) le plus grand nombre d'adhérents - ne pouvait donc constituer sa propre RSE et bénéficier des droits garantis, selon le système juridique italien, aux représentants syndicaux présents sur les lieux de travail

En outre, Fiat a transféré son usine de Pomigliano d'Arco à la société Fabbrica Italia Pomigliano s.p.a., entreprise contrôlée à 100 \% par Fiat s.p.a. ; lorsque les anciens salariés de Fiat ont été réembauchés, aucun adhérent de la FIOM n'a été retenu, ce qui a entraîné une diminution draconienne du nombre d'adhérents à ce syndicat. Par ailleurs, Fiat a refusé de procéder à la retenue de la cotisation syndicale sur le salaire des adhérents de la FIOM, ce qui a ainsi compliqué le règlement des adhésions pour le syndicat.

À l'usine de Melfi, Fiat a licencié trois délégués syndicaux, accusés d'avoir empêché, dans le cadre d'une grève, la poursuite de l'activité de production.

À l'occasion de la rencontre avec la délégation spéciale de négociation en vue de la constitution du comité d'entreprise européen (CEE), Fiat Industrial s.p.a. a réclamé, pour finir, le remplacement du membre désigné par la FIOM, au motif que ce syndicat n'avait pas signé la CCSL.

Les décisions sommairement décrites plus haut ont été systématiquement contestées par la FIOM devant les juges du travail.

C'est dans le cadre de cette stratégie judiciaire qu'une plainte a été présentée, le 31 Mai 2012, auprès du Comité de la liberté syndicale de I'OIT, alors même que les questions de légitimité constitutionnelle n'avaient pas encore été soulevées devant la Cour constitutionnelle (celles-ci ont été soulevées le 04 Juin 2012 par le Tribunal de Modène, le 25 septembre 2012 par le Tribunal de Verceil, le 28 Novembre 2012 par le Tribunal de Melfi et le 12 Décembre 2012 par le Tribunal de Turin).

\section{II - LA PLAINTE PRÉSENTÉE AU COMITÉ DE LA LIBERTÉ SYNDICALE, LES CONCLUSIONS DU COMITÉ ET LEUR SUIVI}

Dans sa plainte présentée au Comité de la liberté syndicale, la CGIL (Confédération générale italienne du travail) reprochait au gouvernement italien d'être " demeuré totalement passif face aux comportements discriminatoires et préjudiciables à la liberté syndicale de la FIOM-CGIL », violant ainsi l'article $1^{\text {er }}$ de la convention $n^{\circ} 98$ de l'OIT qui attend des gouvernements qu'ils exercent une surveillance stricte et entreprennent des actions efficaces contre toute forme de discrimination syndicale.

La CGIL reprochait également au gouvernement la violation de l'article $1^{\text {er }}$ de la convention $n^{\circ} 135$ de l'OIT, étant donné que celui-ci ne prenait pas toutes les mesures requises pour garantir aux représentants syndicaux une protection effective contre toute action visant à porter atteinte à leurs fonctions. La violation de l'article 2 de la convention était également invoquée, car les représentants de la FIOM ne pouvaient jouir des droits prévus pour les représentants syndicaux dans le Statut des travailleurs.

Enfin, il était reproché au gouvernement italien de violer l'article 2 de la convention $n^{\circ} 87$, car il permettait à Fiat de traiter différemment les organisations syndicales sur la base de leur seule décision de signer ou non la convention collective (c'est-à-dire que seules celles qui signaient la convention collective pouvaient constituer une RSE au sein des usines Fiat). 
Dans les conclusions adoptées lors de sa 320ème session (13-27 mars 2014) ${ }^{6}$, le Comité de la liberté syndicale commence par affirmer que l'arrêt n²31/2013 de la Cour constitutionnelle, intervenu entre-temps, promeut «le respect des conventions et principes de l'OIT en matière de liberté syndicale et de négociation collective, dans la mesure où subordonner la possibilité de disposer d'une représentation syndicale d'entreprise à l'obtention d'un accord avec l'employeur sur le contenu d'une convention collective pourrait être susceptible de restreindre la liberté d'action des organisations syndicales ainsi que la liberté de négociation collective » (§ 619). II convient d'observer que ce n'est qu'à la suite de l'arrêt de la Cour constitutionnelle que Fiat s'est souciée de faire parvenir au Comité ses propres observations, en signalant que le groupe avait enfin accordé à la FIOM le droit de constituer une RSE dans chaque usine Fiat.

Néanmoins, le Comité rappelle que l'arrêt de la Cour constitutionnelle n'aborde pas le problème plus général du défaut d'une loi sur la représentativité syndicale et ne détermine pas de critère sélectif de la représentativité syndicale permettant la reconnaissance des droits syndicaux renforcés prévus par le Statut des travailleurs en cas d'absence de convention collective applicable au sein de l'entreprise ou de l'impossibilité d'aboutir à un accord collectif d'entreprise (Cour const., arrêt n²31/2013, § 9). C'est pourquoi, le Comité demande au gouvernement d'agir au plus vite sur cette question et de le tenir informé des initiatives qui seront prises ( $\S 620$ ).

Le Comité demande ensuite au gouvernement de garantir que tous les salariés du groupe affiliés à la FIOM-CGIL puissent continuer à voir leurs cotisations déduites de leurs salaires et versées à ladite organisation syndicale (§ 621).

Le Comité demande aussi d'indiquer si, en exécution de l'arrêt nº 18368/2013 de la Cour de cassation, les trois délégués syndicaux ont été effectivement réintégrés à l'usine de Melfi (§ 624).

Soulignant que «la discrimination antisyndicale constitue l'une des violations les plus graves de la liberté syndicale ", le Comité demande enfin à être informé des décisions judiciaires encore en attente concernant la constitution du Comité d'entreprise européen et les discriminations visant les adhérents à la FIOM de l'usine de Pomigliano, en invitant le gouvernement à prendre les initiatives nécessaires pour empêcher que de nouveaux conflits de nature similaire ne surviennent au sein du groupe Fiat (§ 625).

Signalons que c'est la première fois que le Comité de la liberté syndicale adopte des recommandations aussi sévères à l'encontre de l'Italie?

Suite à cette décision du Comité, un bref dialogue s'est engagé avec le gouvernement italien qui, le 24 Juillet 2014 et le 17 Septembre 2014, a présenté des informations sur le suivi du cas. La communication du gouvernement contient aussi les observations de Fiat (datant du 7 Juillet 2014 et du 8 Août 2014) ainsi que celles de la CISL (Confédération italienne des syndicats de travailleurs) et de I'UIL (Union italienne du travail) (datant du 15 Juillet 2014). Les documents du gouvernement n'ont pas été transmis à la CGIL qui avait déjà présenté, le 16 Juillet 2014, ses propres observations concernant l'affaire (observations annexées par ailleurs à la première communication du gouvernement). La plaignante n'a donc pas pu répondre sur ce point. D'autre part, dans leur communication, la CISL et l'UIL

$6371^{\text {ème }}$ Rapport du Comité de la liberté syndicale, cas n²953.

7 V. Bavaro, "L'Oil e il reclamo della Cgil (a proposito del caso Fiat) », II diario del lavoro, 14 Avril 2014. 
regrettent de ne pas avoir eu la possibilité de présenter leurs considérations au cours de la phase qui a précédé les conclusions du Comité de liberté syndicale.

Il convient de noter que le Comité de la liberté syndicale institué par l'OIT peut informer les plaignants du contenu des observations du gouvernement, en les invitant à présenter à leur tour leurs propres observations sur la question. De même, la Direction générale, à la lumière des observations du gouvernement, peut estimer nécessaire d'obtenir des informations supplémentaires de la part des plaignants, en leur demandant de communiquer leurs réponses quant à la position du gouvernement. II n'est donc pas garanti aux parties concernées qu'elles puissent accéder librement aux documents déposés par le gouvernement et les parties adverses.

Au sujet de la représentativité syndicale, le gouvernement se limite à mentionner l'accord interconféderal sur les représentants syndicaux signé le 10 Janvier 2014 par la CGIL, la CISL, I'UIL et la Confindustria (Confédération italienne des entreprises), sans expliquer pour autant que cet accord ne s'applique pas au cas Fiat, du moment que Fiat ne fait pas partie de Confindustria. Le choix du gouvernement est sans aucun doute conditionné par la position de la CISL et de I'UIL, qui indiquent que les situations conflictuelles ayant donné naissance au cas Fiat «ont bénéficié d'une conciliation et d'un règlement plus que suffisants à travers les mécanismes de protection ordinaires largement proposés par le droit interne, sans qu'il y ait besoin, à cette fin, de demander au gouvernement des interventions qui ne pourraient que revêtir le caractère d'une ingérence abusive dans les principes de liberté et d'autonomie associative que le Comité a officiellement pour but de défendre ».

Pour ce qui a trait à la déduction des cotisations syndicales, le gouvernement rappelle la position de Fiat : I'obligation de retenue sur les salaires des travailleurs prévue dans la convention collective du groupe Fiat ne s'applique qu'aux seuls syndicats signataires de cette convention; tous les autres syndicats peuvent percevoir les cotisations de leurs affiliés directement sur le lieu de travail ou leur demander d'effectuer un versement sur un compte courant bancaire. Le gouvernement ajoute aussi qu'afin de ne pas alimenter le contentieux, les groupes Fiat et Fiat Industrial ont accepté, dès le mois de septembre 2012, d'effectuer les retenues pour le compte de la FIOM.

Quant au licenciement des trois délégués syndicaux à l'usine de Melfi, le gouvernement signale le caractère "litigieux " de l'affaire judiciaire sans même mentionner que les travailleurs ont été effectivement réintégrés (à l'exception de l'un d'entre eux, qui bénéficiait d'un congé pour exercer une fonction politique).

Relativement aux discriminations syndicales à l'encontre des affiliés à la FIOM au sein de l'usine de Pomigliano, le gouvernement joint le procès-verbal de conciliation en date du 30 Mai 2014, dans lequel Fiat s'engage à réembaucher les travailleurs victimes des discriminations et où la FIOM (en son nom propre et pour le compte des travailleurs) déclare qu'elle n'a plus rien d'autre à exiger et s'engage, en ce qui concerne ces faits et ce contentieux, à n'intenter aucune autre action à l'encontre de Fiat.

Le gouvernement italien n'évoque pas la constitution du CEE, au sujet de laquelle le Groupe Fiat Industrial signale, dans une lettre, que la décision de la Cour d'appel de Turin du 07 Mai 2014 est de nature " purement déclaratoire et, en tant que telle, sans effets substantiels et dépourvue de dispositions concrètes à l'encontre de l'entreprise ». En réalité, la Cour d'appel de Turin a réformé le jugement du Tribunal de Turin en date du 20 Février 2013, en affirmant le caractère antisyndical du comportement de Fiat Industrial s.p.a lorsque celle-ci s'est immiscée dans la désignation de la délégation spéciale de négociation et a 
exigé le remplacement du membre affilié à la FIOM. Le diktat de l'entreprise - affirment les juges turinois - constitue « une restriction directe et immédiate de la liberté et de l'activité syndicale et crée une discrimination évidente au détriment du syndicat demandeur ».

Étant donné les informations reçues du gouvernement, le Comité de la liberté syndicale a décidé, en juin 2017, de ne pas poursuivre l'examen de ce cas. Dans son suivi, le Comité mentionne notamment la position de la CISL et de I'UIL et note " avec intérêt » que la question de la représentativité syndicale est résolue par l'accord interconfédéral sur les représentants syndicaux ${ }^{8}$.

\section{Le rideau tombe... mais les problèmes restent à résoudre}

L'épilogue du cas Fiat laisse indubitablement perplexe. L'affaire a été classée sans suite alors que le gouvernement n'a rien fait pour garantir «le libre exercice du droit syndical » (art. 11 de la convention $n^{\circ} 87$ de l'OIT). Plus particulièrement, la question de la représentativité syndicale demeure non résolue, du moins pour les entreprises non adhérentes à la Confindustria, auxquelles l'accord interconféderal sur les représentants syndicaux ne s'applique pas. Par ailleurs, dans les conclusions et le suivi du Comité de la liberté syndicale, l'art. 8 de la loi n 148/2011 n'est jamais cité, alors que la conformité de cette disposition aux conventions de l'OIT a été mise en doute par la CGIL dans sa plainte?.

Les événements qui se sont récemment déroulées dans les usines italiennes de Fiat démontrent que les questions soulevées devant le Comité de l'OIT ne sont pas totalement résolues. Sans prétendre à l'exhaustivité, il suffit de rappeler que Fiat se refuse à convoquer une table de négociation comprenant les représentants de tous les syndicats car la FIOM n'a jamais signé la convention de groupe. Le groupe Fiat n'applique pas la convention collective nationale du travail de la branche métallurgie signée par tous les syndicats fin 2016, même si cette dernière contient des dispositions constituant une amélioration pour les travailleurs. Ainsi, les dispositions de la convention de groupe (par exemple, en ce qui concerne l'organisation du travail) s'appliquent à tous les travailleurs ${ }^{10}$. De plus, la convention de groupe est la seule convention collective à laquelle Fiat se réfère lorsque la loi fait renvoi aux conventions collectives.

En mars 2016, Fiat et les syndicats signataires de la convention de groupe sont parvenus à un accord sur l'utilisation du contrat de solidarité conformément à l'art. 21 alinéa 5 du décret législatif $n^{\circ} 148 / 2015^{11}$. Même cet accord a été signé en excluant les RSE de FIOM et la FIOM elle-même. Et les contrats de solidarité sont applicables à tous les travailleurs (même ceux affiliés à la FIOM).

Le 11 Juillet 2016, l'accord visant à la reconstitution du CEE de Fiat a été signé par Fiat Chrysler Automobiles, IndustriAll European Trade Union et les syndicats italiens de la métallurgie (inclus la FIOM), il ne semble pas que la réglementation interne sur les CEE

8 Effect given to the recommendations of the committee and the Governing Body, Report $n^{\circ} 382$, June 2017, case no. 2953 (Italy), §104 et 105.

9 L'art. 8 de la loi n 148/2011 permet aux conventions collectives d'entreprise de déroger à la loi ou aux conventions collectives nationales, sans spécifier les critères de représentativité que les syndicats doivent respecter pour signer tels accords.

10 Sur ce point, voir dernièrement, Cass., arrêt n² 27115/2017.

11 Le contrat de solidarité permet à l'employeur de réduire l'horaire de travail des travailleurs en cas de crise de l'entreprise. 
respecte la directive $n^{\circ} 2009 / 38$ ainsi que les conventions de l'OIT $n^{\circ} 87$ et $n^{\circ} 135$. Dans plusieurs dispositions, la loi $n^{\circ} 113 / 2012$ se réfère en effet aux « organisations syndicales qui ont signé la convention collective nationale s'appliquant à l'entreprise ou au groupe d'entreprises concernées » (art. 5 al. $1 ; 6$ al. 2 et $3 ; 7$ al. 2 ; 9 al. 6 et 7 ; 15 al. 1-a); 16 al. 3 et 6 ). Comme le démontre le cas Fiat, il est fort possible que l'entreprise (ou le groupe) n'applique aucune convention collective nationale. Et il est aussi possible que la convention collective nationale appliquée par l'entreprise (ou le groupe) n'ait pas été signée par un syndicat qui, bien que représentatif, serait ainsi privé de la possibilité de participer à la constitution du CEE.

Ajoutons à ceci qu'aucun des jugements rendus par les Cours nationales après les conclusions du Comité de la liberté syndicale ne cite les recommandations de ce dernier. La réglementation de l'OIT et la jurisprudence du Comité n'ont même pas été envisagées comme des arguments ad adjuvandum par les juges qui ont décidé sur l'obligation de l'employeur de verser les contributions syndicales des travailleurs adhérents de la FIOM, bien que le Comité ait expressément rappelé que «la suppression de la possibilité de retenir les cotisations à la source, qui pourrait déboucher sur des difficultés financières pour les organisations syndicales, n'est pas propice à l'instauration de relations professionnelles harmonieuses et devrait donc être évitée» $(\S 621)^{12}$. Les recommandations du Comité de la liberté syndicale ne sont pas davantage mentionnées dans l'arrêt du 07 Mai 2014 de la Cour d'appel de Turin à propos du CEE de Fiat, laquelle s'est exprimée quelques mois après ce dernier.

Dans le cas Fiat, le dialogue multi-niveaux n'a donc pas garanti une protection effective et complète des droits syndicaux. D'un côté, la faute en incombe aux problèmes de procédure déjà signalés : les règles procédurales de I'OIT ne garantissent pas le respect du contradictoire ; cela non seulement constitue une entrave aux droits de la défense des parties, mais empêche aussi le Comité d'avoir une vision complète de l'affaire, en disposant de toutes les informations nécessaires pour s'exprimer sur le litige. Dans le cas Fiat, les mésententes entre les confédérations syndicales n'ont pas été propices à la garantie des droits syndicaux. Mais c'est justement la rupture de l'unité syndicale qui rend encore plus urgente l'intervention du gouvernement pour empêcher que de nouveaux conflits surviennent (comme cela est demandé par le Comité de la liberté syndicale au § 625 de ses conclusions).

Le silence des Cours nationales sur les recommandations du Comité de la liberté syndicale et, en général, sur la réglementation de l'OIT est aussi un sujet de préoccupation. Les conventions de l'OIT sont rarement mentionnées par les Cours italiennes et les décisions des comités de contrôle de l'OIT ne sont jamais citées. Dans la majorité des arrêts, les conventions de l'OIT sont mentionnées afin d'argumenter ad adjuvandum une décision déjà prise sur la base du droit interne ${ }^{13}$. Cependant, l'efficacité des conventions de I'OIT, au-delà de leur valeur interprétative, demeure encore obscure.

12 Voir dernièrement, Cass., arrêt n 5321/2017.

13 Récemment, la Cour constitutionnelle a mentionné les conventions de l'OIT et de la CSE pour justifier l'interprétation évolutive du texte constitutionnel (Cour constitutionnelle, arrêt n 178/2015, cité par la Cour de Cass., arrêt n² 21232/2015). 


\section{II - GRÈVE ET PROCÉDURES D'URGENCE : LE C.E.D.S. ET LE JUGE BELGE}

Le droit de grève s'est développé en Belgique dans le silence de la loi. Sa reconnaissance pleine et entière découle d'un arrêt historique de la Cour de cassation datant de $1981^{14}$, sur la base d'un raisonnement a contrario tenu à la lumière de la loi relative aux prestations d'intérêt public en temps de paix ${ }^{15}$.

La Constitution belge ne mentionne pas la grève, même après la réforme de 1993 qui a inséré une série de droits sociaux, économiques et culturels dans l'article 23 de la Constitution. La consécration législative (indirecte) n'arrivera qu'en 1990 avec la loi de ratification de la CSE. C'est pour cette raison que ce document remplit, pour le droit de grève en Belgique, une fonction quasi-constitutionnelle puisqu'il est souvent mentionné par les décisions jurisprudentielles en matière de grève et d'actions liées à la grève ${ }^{16}$. L'article 6(4) de la Charte est considéré en effet par la doctrine et la jurisprudence comme ayant un effet direct ${ }^{17}$.

Vers la fin des années 80 , les employeurs ont commencé à présenter des recours par la voie de procédures d'urgence (ce que l'on appelle des actions en référé) pour attaquer ce que l'on qualifie d'« actes détachables » de l'action de grève. Ces actes « détachables » concernent tous les comportements qui dépassent le cadre du simple arrêt du travail et visent notamment l'activité de mise en place de piquets de grève et l'occupation éventuelle des locaux de l'entreprise. Une telle procédure d'urgence se déroule devant le président du tribunal de première instance (c'est-à-dire le juge civil) et se fonde sur l'article 584 du Code judiciaire belge, qui permet à quiconque pouvant prouver qu'il a personnellement et directement intérêt de recourir au juge pour obtenir la préservation d'un intérêt protégé menacé à l'occasion d'un conflit collectif. Les intérêts protégés invoqués par les employeurs dans ce contexte concernent le droit de propriété, ainsi que la liberté du commerce et de l'industrie ${ }^{18}$.

Si le président du tribunal considère que l'apparence de bien-fondé de la demande (fumus boni iuris) est suffisante, il rend une ordonnance ${ }^{19}$ accompagnée d'une astreinte, c'est-à-dire une sanction pécuniaire en cas de non-respect de l'interdiction contenue dans ladite astreinte. En outre, le juge doit prendre en considération l'existence de la "nécessité absolue $»^{20}$ qui s'appuie, en règle générale, sur la base de l'argument selon lequel il serait impossible à l'employeur de connaître, en amont, quels travailleurs vont participer aux piquets de grève.

14 Cass., arrêt du 21.12.1981, Journal des tribunaux du travail, 1982, 329.

15 Loi relative aux prestations d'intérêt public en temps de paix, 19.08.1948.

16 Voir par exemple Cour du travail de Bruxelles, 05.11.2009, RG 2009/AB/52381.

17 En plus, selon la jurisprudence de la Cour de cassation le droit international ayant un effet direct prime sur les dispositions nationales. Voir Cass. 27 mai 1971, Pasicrisie, 1971, I, p.886.

18 Consacrée par ce que l'on a coutume d'appeler le " décret d'Allarde » (il s'agit en fait d'une loi) des 2 et 17 mars 1791.

19 Dans la grande majorité des cas, les ordonnances rendues dans le cadre de cette procédure ne sont pas publiées. Les auteurs tiennent à remercier Jean-François Neven et Filip Dorssemont de leur avoir fourni une aide précieuse pour retrouver la trace des nombreuses ordonnances nécessaires à la rédaction du présent article.

20 La présence de l'absolue nécessité est en effet nécessaire pour l'introduction de la procédure par requête unilatérale. 
Les présidents des tribunaux belges - bien qu'ayant des démarches peu uniformes à cet égard - soient régulièrement intervenus dans les questions relatives à l'exercice du droit de grève, dans le cadre de mesures d'urgence visant à interdire le blocage, par les syndicats, de l'accès au lieu de travail aux travailleurs non-grévistes. Les juges ont agi, inaudita altera parte, à la demande de l'employeur et la plupart du temps, la requête se fondait sur la simple annonce - diffusée par voie de presse ou par tract $^{21}$ - de la mise en place de piquets de grève ou de la décision de bloquer l'accès à l'entreprise. L'ampleur de ces mesures est extrêmement variable, tout autant sur le plan des modalités d'interdiction que du point de vue de la période couverte (de 24 heures à plusieurs mois).

La possibilité pour les syndicats de présenter un recours en tierce opposition contre ces décisions s'est avérée être une arme véritablement émoussée ${ }^{22}$. En premier lieu, parce que souvent les syndicats n'étaient même pas au courant des décisions du juge et que ces dispositions n'étaient opposées aux grévistes qu'au moment de l'action collective. De la sorte, les ordonnances de référé avaient pour résultat d'empêcher le déroulement de la protestation des travailleurs, brisant l'élan de cette dernière puisque la présentation de la tierce opposition devrait, de toute manière, intervenir après ${ }^{23}$. En second lieu, parce que les juges d'appel ont souvent considéré qu'une fois que le conflit collectif faisant l'objet de la mesure d'urgence serait réglé, la tierce opposition serait irrecevable pour défaut d'intérêt à agir et absence d'objet ${ }^{24}$. Cette approche a été longuement confortée par la jurisprudence de la Cour de cassation ${ }^{25}$.

La Cour est récemment revenue sur la question, en modifiant sa jurisprudence et en admettant la possibilité d'interjeter appel contre les mesures prononcées par le juge des référés, même lorsque le conflit collectif a désormais cessé ${ }^{26}$. Ce contrôle se contente de vérifier les apparences de droit des mesures concernées, de sorte que le juge des référés ne puisse être sanctionné qu'en cas d'excès de pouvoir, après avoir procédé à une application déraisonnable des droits des parties ou refusé de manière déraisonnable l'application de ces droits ${ }^{27}$. La violation des dispositions de droit matériel demeure insignifiante en cas de tierce opposition. Le contrôle exercé par la Cour de cassation revêt donc un caractère marginal.

21 J-F. Neven, « Piquets de grève : les suites de la décision du Comité européen des droits sociaux du 13 septembre 2011 », in C. Guillain, F. Dorssemont, I. Ficher, Droit de grève : actualités et questions choisies, Bruxelles, Larcier, 2015, p. 46.

22 F. Dorssemont, «Libres propos sur la légitimité des requêtes unilatérales contre l'exercice du droit à l'action collective à la lumière de la décision du Comité européen des droits sociaux », in I. Ficher et autres (dir.), Actions orphelines et voies de recours en droit social, Limal, Anthemis, 2012, p. 132.

23 P. Humblet, «Behoort de tussenkomst van de rechter in collectieve conflicten tot het verleden? », in Rechtspraak Antwerpen Brussel Gent, 2013, p. 860.

24 Civ. Bruxelles, 4.2.1994, J.L.M.B., 1994, p. 657 ; Journal des tribunaux du travail, 1994, p. 331 ; civ. Liège (Prés.), 30.7.1999, Journal des tribunaux du travail, p. 377 ; contre civ. Bruxelles (Prés.), 8.3.2000, Journal des tribunaux du travail, 2000, p. 208 ; Mons, 21.11.2005, Journal des tribunaux du travail, 2006, p. 26.

25 Cass., 17.4.2009, Journal des tribunaux, 2009, p. 672. Dans le même sens, Cass., 19.1.2006, Pasicrisie, I, p. 188 ; Cass., 19.9.2002, Pasicrisie, I, p. 1697 ; Cass., 9.6.2000, Pasicrisie, I, p. 1051.

26 Cass., 4.2.2011, R.G. $n^{\circ}$ C.10.0459.N, Journal des tribunaux, 2011, p. 246. Voir J-F Neven, « Les piquets de grève, la procédure sur requête unilatérale et les pouvoirs du juge des référés après la décision du Comité Européen des Droits Sociaux du 13 septembre 2011 », Revue de droit social, 4, 2012, p. 407.

27 Cass., 31.1.1997, Pasicrisie, I, p. 56. 
Par le passé, le juge des référés n'a pas été sanctionné tant qu'il s'est limité à vérifier l'apparence de bien-fondé de la demande (fumus boni iuris) sans tenir compte des autres droits matériels qui ne pourraient pas raisonnablement avoir un impact sur les mesures provisoires demandées ${ }^{28}$.

\section{A - LA DÉCISION DU C.E.D.S.}

La pratique des tribunaux belges a fait l'objet d'un recours collectif devant le C.E.D.S. ${ }^{29}$, présenté par l'ensemble des syndicats les plus représentatifs (CSC/ACV, FGTB/ABVV et CGSLB/ACLVB) et par la Confédération européenne des syndicats.

Il est important de souligner que les syndicats belges avaient " préparé le terrain ", en transmettant des observations à l'occasion des précédentes séries de conclusions du C.E.D.S. ${ }^{30}$.

Dans sa décision, le C.E.D.S. a clarifié tout d'abord le fait que le droit de grève, protégé par l'article 6(4) de la CSE, inclut « non seulement le droit de cesser le travail mais aussi, entre autres, celui de participer à des piquets ${ }^{31}$. Mais le Comité a bien pris soin de distinguer les piquets qui, à travers des intimidations ou des violences, portent atteinte à la liberté des travailleurs non-grévistes de ne pas prendre part à la grève, lesquels ne sont pas inclus dans l'article 6(4)32, et les piquets qui, en revanche, n'entravent pas le libre choix de participer ou non à la grève et qui sont protégés par ce même article ${ }^{33}$. En ce qui concerne les non-grévistes, il semble donc que le droit protégé par l'article soit celui de pouvoir décider s'il convient de prendre part ou non à la grève et non pas celui d'accéder à l'entreprise et/ou de pouvoir effectuer son travail ${ }^{34}$.

Une fois qu'il a été établi que les piquets s'inscrivent bien dans le cadre du droit d'action collective et donc de l'article 6(4), le C.E.D.S. procède à l'analyse des restrictions imposées à ce dernier par la mise en œuvre des procédures sur requête unilatérale. L'élément le plus important de la décision du C.E.D.S. concerne l'aspect procédural de la situation belge. À ce propos, le Comité a constaté que la jurisprudence belge ne présentait pas les conditions d'une stabilité suffisante permettant de garantir la sécurité juridique des parties concernées et, plus particulièrement, la possibilité, pour les travailleurs souhaitant organiser un piquet de grève, de savoir par avance si ce dernier fera l'objet de restrictions légales ${ }^{35}$. En outre, le Comité affirme que la nature unilatérale de la procédure présente le

28 Cass., 25.11.1996, R.G. n S.960030.N ; Cass., 25.4.1996, R.G. n C.930356.N ; Cass., 25.6.1999, R.G. ${ }^{\circ}$ C.970372.N ; Cass., 5.5.2000, R.G. $n^{\circ}$ C.99.0013.N ; Cass., 2.6.2006, R.G. $n^{\circ}$ C.030211.F ; Cass. 31.1.1997, Pasicrisie, I, p. 56.

29 C.E.D.S., décision du 13.9.2011 sur la plainte collective n. 59/2009, Ces, Cgslb, Csc, Fgtb/Belgique.

30 C.E.D.S., Conclusions XVI-1 - Belgique - art. 6(4).

31 C.E.D.S., décision du 13.9.2011 sur la plainte collective $n^{\circ}$ 59/2009, § 29.

32 Ibidem, § 35 .

33 Ibidem, § 36.

34 F. Dorssemont, cit. p. 140-141. Pour une opinion divergente sur le sujet, voir D. Dejonghe, F. Raepsaet, "L'intervention du juge des référés sur requête unilatérale en cas de conflit collectif : contraire à la Charte sociale européenne ? "Revue de droit social / Tijdschrift voor sociaal recht, 2013, p. 143.

35 Ibidem, § 43. 
risque que les intérêts légitimes de l'une des parties ne soient pas pris en compte et, en conséquence, ne respectent pas l'exigence « d'équité procédurale », implicite - selon le raisonnement du C.E.D.S. - dans la condition suivant laquelle les restrictions doivent être « prévues par la loi ». L'exclusion des syndicats de la procédure, ainsi que la nécessité qui s'ensuit pour ces derniers de n'intervenir que lorsqu'une décision contraignante a déjà été prise par le juge, sont donc considérées comme enfreignant cette impartialité procédurale et constituant une violation de l'article G de la $\mathrm{CSE}^{36}$.

\section{B - L'APPLication PAR les Cours nationales}

Suite à cette décision, le gouvernement belge s'était engagé devant le Conseil des ministres à informer la justice de ladite décision. Le gouvernement a donné suite à cet engagement en deux temps : en faisant tout d'abord connaître la décision au Collège des procureurs généraux le 3 janvier 2013, puis au Collège provisoire des cours et tribunaux le 16 septembre 2014. Ce dernier organe a par ailleurs été chargé de communiquer l'information aux présidents de cours et de tribunaux qui, comme nous l'avons vu, sont directement impliqués dans les procédures de référé.

Dans son évaluation de 2015, le C.E.D.S. a confirmé que la situation de la Belgique n'avait pas changé et que la violation de l'article 6(4) de la CSE perdurait ${ }^{37}$. La situation devra être reconsidérée dans le cadre de la prochaine série de conclusions. À ce sujet, le gouvernement belge a adressé son rapport national au C.E.D.S. Dans ce rapport, le gouvernement belge considère que la situation a été rendue conforme à la décision du C.E.D.S. et de la CSE, sur la base de quelques éléments principaux. Le gouvernement fait notamment référence à certaines décisions jurisprudentielles qui ont intégré la décision du C.E.D.S., en soulignant par ailleurs que les juridictions ont accordé plus d'importance au respect du contradictoire, que les juridictions d'appel ont accepté d'envisager la légalité des mesures de contrôle même dans le cas où le conflit collectif a cessé et affirme enfin que les partenaires sociaux ont tenté de négocier un gentlemen agreement au niveau interprofessionnel relatif à l'exercice du droit de grève (négociation qui, entre-temps, a échoué).

Parallèlement à cette description, qui est bien entendu centrée sur les évolutions positives, l'on ne peut pas ne pas relever que certains présidents de tribunal continuent à accorder des mesures d'urgence sans se référer à la décision du C.E.D.S. et sans en tenir compte, que ce soit de manière implicite ou explicite. II ne semble pas que ces mesures aient pris en compte les enseignements de la décision puisqu'elles n'excluent pas du champ des interdictions prononcées, par exemple, les piquets de grève pacifiques effectués sans bloquer totalement l'accès à l'entreprise.

À deux reprises, le président du tribunal de Tournai a accordé des mesures d'urgence et interdit la mise en place de piquets de grève. Ceci, dans le premier cas, parce que des informations rapportées par la presse semblaient sous-entendre que les organisations syndicales souhaitaient bloquer l'accès à l'usine ${ }^{38}$; dans le second cas, parce que cette volonté avait été clairement explicitée par les organisations elles-mêmes dans le tract qui

36 Ibidem, § 44. La décision évoque en effet aussi le fait que la procédure sur requête unilatérale contraint les organisations syndicales à introduire une lourde procédure en appel, critique qui semble alors porter plutôt sur la proportionnalité de la restriction.

37 C.E.D.S., Évaluation du suivi, plainte collective $n^{\circ}$ 59/2009, 4.12.2015.

38 Prés. Civ. Hainaut, division Tournai, 21.11.2014, R.G. 14/950 B, inédite. 
annonçait la grève ${ }^{39}$. Dans les deux cas, le juge des référés n'a pas envisagé la question sous l'angle de l'existence ou du risque d'intimidations et/ou de violences. Le président du tribunal de Charleroi, en revanche, a choisi de se référer à la décision du C.E.D.S., en donnant toutefois une interprétation particulièrement restrictive de celle-ci, de sorte que le simple fait d'occuper en groupe (même pacifiquement) l'entrée de l'entreprise, sans pour autant en bloquer l'accès, a été considéré comme une forme d'intimidation in re ipsa à l'égard des non-grévistes et ne se trouve donc pas protégé par le droit de grève ${ }^{40}$.

Une ordonnance de référé prononcée par le président du tribunal de Bruxelles, qui avait accordé des mesures de contrôle, a été annulée suite à la tierce opposition formée par le syndicat. C'est à travers cette opposition qu'il est alors apparu que les travailleurs auxquels le piquet de grève interdisait l'entrée de l'usine avaient été, en réalité, embauchés par une agence intérimaire en réaction à la grève (et donc, en violation de la loi $)^{41}$.

Inversement, d'autres juridictions ont commencé à se montrer plus prudentes, sans faire toutefois référence à la décision du C.E.D.S. C'est le cas de la Cour d'appel de Mons, qui a critiqué la décision en référé sur la base du constat qu'un préavis de grève avait été déposé quelques semaines avant son déroulement effectif, situation qui aurait permis en plus du fait que l'identité des représentants des syndicats organisateurs était connue - l'instauration d'une procédure respectant le principe du contradictoire ${ }^{42}$.

Plus récemment, la Cour d'appel de Bruxelles ${ }^{43}$ a annulé une ordonnance rendue par le tribunal de première instance en 2015 dans le contexte d'une grève organisée dans le secteur ferroviaire. La société gérant le réseau ferroviaire avait demandé et obtenu du président du tribunal de Bruxelles une ordonnance empêchant quiconque d'avoir accès aux cabines de contrôle des aiguillages, d'occuper les voies et d'utiliser des fumigènes rouges à proximité de celles-ci. L'ordonnance était justifiée par la nécessité de préserver la sécurité des tiers et des grévistes eux-mêmes. Un travailleur - qui plus est, représentant syndical avait enfreint cette interdiction et s'était vu infliger une amende de 1000 euros. Le juge d'appel a annulé l'ordonnance et l'amende en se fondant sur la possibilité d'engager une procédure contradictoire, au vu du préavis de grève déposé un mois avant le déroulement de celle-ci.

Suite à cette décision, la société gérante, dans le cadre d'une grève ultérieure, a assigné en justice le secrétaire général de l'un des syndicats organisateurs de l'action collective. Le président du tribunal de Bruxelles a refusé les mesures de contrôle en vertu du constat que le secrétaire général ne pouvait être considéré comme le représentant légal des personnes susceptibles de tenter concrètement de bloquer les voies lors de la grève ${ }^{44}$. Dans le même

39 Prés. Civ. Hainaut, division Tournai, 2 avril 2014, R.G. 14/293/B, inédite.

40 Prés. Civ. Charleroi, 21 janvier 2014, RG n 13/2754/A, inédite

41 Prés. Civ. Brussel, 8 septembre 2014, RG n²014/423/C, inédite.

42 Cour d'appel de Mons, 21 ème ch., 28 novembre 2012, Chronique de droit social, 2014, p. 20 et Journal des tribunaux du travail, 2013, p. 57. Dans le même sens, voir aussi Cour d'appel d'Appel Anvers, 8ème ch., 29 juin 2012, Chronique de droit social, 2014, p. 42.

43 Cour d'appel de Bruxelles, $2^{\text {ème }}$ ch., 15 septembre 2017, inédite.

44 Prés. Civ. Bruxelles, 5 octobre 2017, inédite. Voir aussi J-F. Neven, «Requêtes en matière de grève : priorité au contradictoire. Commentaire de l'arrêt de la Cour d'appel de Bruxelles du 15 septembre 2017 », Terralaboris.be, p. 8, http://www.terralaboris.be//MG/pdf/lb 050 article jfneven req greve.pdf ; ainsi que I. Van Hiel, « Nog eens over de stakingsposten », Chronique de droit social / Sociaalrechtelijke kronieken, 2014, p. 30-34. 
temps, le juge a rappelé que l'entreprise ne pouvait exiger du tribunal d'interdire " à quiconque » d'occuper les voies tout en citant exclusivement le nom d'une personne, sous peine de créer une confusion entre la procédure unilatérale et la procédure contradictoire.

Face à cette cacophonie de décisions, le travail du C.E.D.S., lors de sa prochaine série de conclusions, s'avérera difficile. Le problème fondamental qui semble poindre est l'absence d'une intervention de "nomofilachia $~^{45}$ susceptible de trancher la question au profit de l'une ou l'autre des positions jurisprudentielles. Ayant limité son contrôle, en ce qui concerne les procédures de référé, aux situations d'excès de pouvoir fondé sur une application déraisonnable des droits des parties et ayant opté pour une évaluation purement marginale des droits matériels en jeu dans ces procédures, la Cour de cassation pourra difficilement remplir sa mission, primordiale dans ce contexte. Pour l'heure, le fait que la Cour suprême belge puisse exercer un contrôle sur les décisions en référé, en enjoignant aux présidents des Cours civiles de respecter les articles 6(4) et G de la CSE ${ }^{46}$, semble donc fort improbable ${ }^{47}$.

Le cas belge fait ainsi ressortir qu'en l'absence d'un interlocuteur placé au sommet des juridictions nationales, qu'il s'agisse d'une Cour constitutionnelle ou d'une Cour de cassation, la protection proposée par le niveau supranational risque de demeurer sans effet concret ou, tout au plus, porteuse d'un effet semblable à la soft law par rapport aux juridictions nationales.

\section{Conclusion}

Les deux cas que nous venons de décrire montrent que la protection multi-niveaux des droits sociaux ne doit pas être envisagée comme une possibilité « d'appel » sui generis à une autorité supérieure, dans le cadre d'une hiérarchie pyramidale classique, mais plutôt comme un mécanisme dans lequel une multiplicité d'engrenages permet de faire apparaître une vision du problème juridique donné s'appuyant sur différents systèmes de valeurs. Mais c'est précisément la nature complexe de cet engrenage qui en constitue la fragilité intrinsèque... car lorsqu'un rouage se grippe, tous les autres se bloquent.

Les deux cas étudiés prouvent que les mécanismes de fonctionnement de l'engrenage sont perfectibles. Qu'il s'agisse des règles procédurales devant les Cours supranationales, de l'efficacité des sources supranationales dans le droit national ou de la manière dont les Cours nationales abordent les contradictions relatives à leur interprétation ou à leur application, la protection multi-niveaux des droits sociaux dépend du franchissement de tous les obstacles pouvant compromettre le bon fonctionnement de l'engrenage.

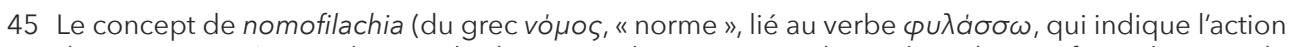
de "protéger ») est utilisé par la doctrine italienne pour indiquer la tâche, confiée à la Cour de Cassation, d'assurer l'interprétation uniforme de la loi par les cours et tribunaux.

46 J-F. Neven, 2012, cit. p. 56-57.

47 En effet, la Cour européenne des droits de l'homme a, dans un contentieux différent, critiqué la position de la Cour de cassation quant au caractère strictement marginal du contrôle exercé sur l'application du droit à l'occasion des procédures en référé. Voy. CEDH, 29 mars 2011, Requête $\mathrm{n}^{\circ}$ 50084/06, RTBf c. Belgique. 